\title{
Statistical Modeling through Analytical and Monte Carlo Methods of the Fat Fraction in Magnetic Resonance Imaging (MRI)
}

\author{
Authors*: Anne M. Calder, Eden A. Ellis, Li-Hsuan Huang and Kevin Park \\ Advisor $^{\dagger}$ : Dr. Angel R. Pineda \\ Department of Mathematics, California State University, Fullerton, CA
}

June 15, 2012

\begin{abstract}
Our project studies the quantification of the uncertainty in fat-fraction estimates using Magnetic Resonance Imaging (MRI). The measured fat fraction is $\frac{|F|}{|F|+|W|}$, where $\mathrm{F}$ is the fat signal and $\mathrm{W}$ is the water signal obtained using MRI. The fat and water signal magnitudes have a Rician distribution. However, the fat fraction has an unknown probability distribution. Knowing the fat-fraction probability distribution will provide us with a better understanding of the uncertainty of fat-fraction estimates used for the diagnosis of liver disease. Our current research focuses on finding the analytic distribution of the fat fraction and numerical simulation using Monte Carlo methods. In the analytic approach, we derived the probability density function of the fat fraction where the fat and water magnitudes follow a normal distribution (restricted to non-negative values) because the normal distribution approximates a Rician distribution for large signal-tonoise ratio (SNR). In the numerical approach, we applied Monte Carlo methods to optimize the fat-fraction estimation, compared analytic with numerical results, and found cases where current estimates of the fat fraction are inaccurate for low SNR.
\end{abstract}

Key Words: MRI, fat fraction, probability, statistics, Monte Carlo

\section{Introduction}

\subsection{Motivation}

In many developed countries, especially the United States, the percentage of the population that is obese is on the rise. A recent study, by the Organization for Economic Cooperation and Development (OECD),

\footnotetext{
*annecalder@csu.fullerton.edu, edenaellis@gmail.com, lihsuan.huang.795@my.csun.edu, kevark@csu.fullerton.edu

†apineda@fullerton.edu

${ }^{\ddagger}$ L. Huang and K. Park are currently at the Mathematics Department of California State University, Northridge, CA.
} 


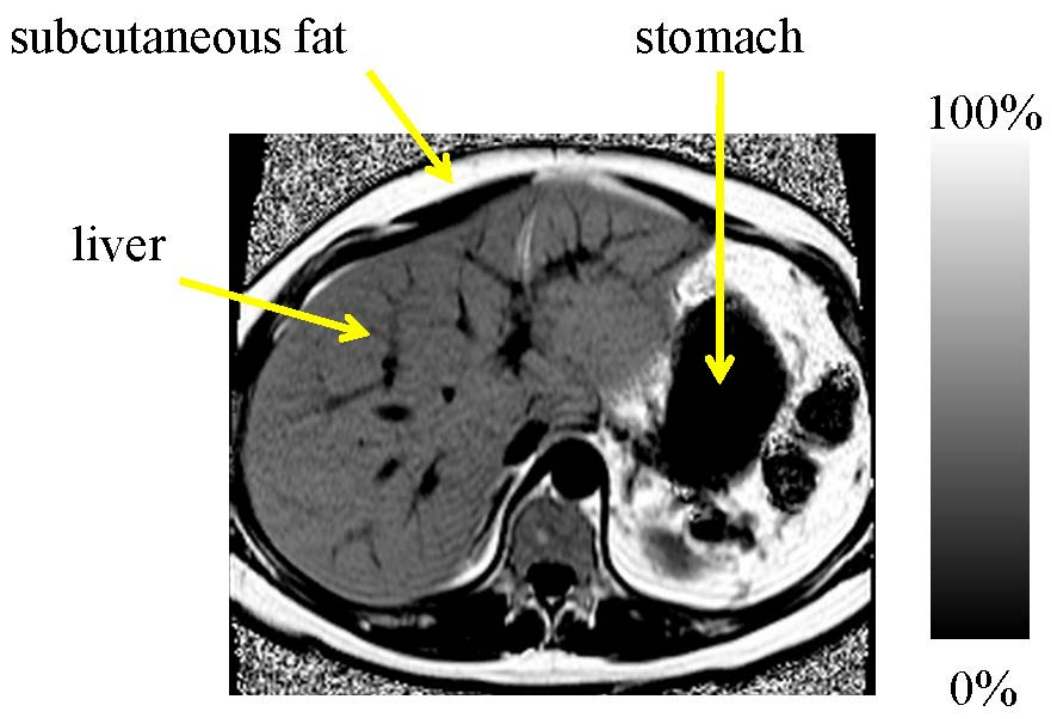

Figure 1: Clinical image of the fat fraction obtained using MRI. This is a patient with a large amount of fat in the liver. A mild case of NAFLD would have a fat fraction of about 10\% [2]; this patient has about $50 \%$. The liver, subcutaneous fat (mostly fat) and stomach (mostly water) are labeled to illustrate the variability of the fat fraction in tissue. Image courtesy of Scott B. Reeder, M.D., Ph.D.

looked at this epidemic, and estimates that by the year 2020, $75 \%$ of Americans will be obese. There are many health problems associated with obesity, in particular, non-alcoholic fatty liver disease (NAFLD), which affects $30 \%$ of adults and $10 \%$ of children in the United States. NAFLD can lead to cirrhosis, hepatocellular carcinoma, and ultimately, liver failure. The current standard for diagnosing NALFD is through a liver biopsy, an invasive procedure that samples a piece of tissue from the liver. However, a biopsy has its limitations, as there is the assumption that the tissue sample that tests $1 / 50,000$ th of the liver is representative of the entire liver. With each sample there is high variability and as a result, the biopsy can give an inaccurate measurement [1].

In addition to its limitations, a biopsy is painful and expensive. This leads to an interest in using a non-invasive method in its place. One method of interest is MRI which uses strong magnetic fields and radio frequency excitation to manipulate the magnetization of some atoms in the body. This is read by a scanner which is recorded into an image. An MRI scan provides good contrast of different tissues in the body including water and fat. Adjusting the parameters, or settings, on the scanner can provide different levels of contrast. A study by Reeder [1] has shown promise in using MRI as a non-invasive method for quantifying fatty liver disease. The way the fat content of a tissue is quantified is through the fat fraction. The current focus of our research is the quantification of the uncertainty of the fat fraction associated with MRI (as shown in Figure 1). 


\subsection{Fat Fraction}

We are defining the measured fat fraction of a volume to be $\widehat{\eta}=\frac{|F|}{|F|+|W|}$, where $\mathrm{F}$ and $\mathrm{W}$ are the complex fat and water signals picked up by the MRI scan. The measurement process generates a signal with real and imaginary components that follow a normal distribution, such that the fat and water measurements follow the model given by:

$$
W=\mu_{W}+\epsilon_{W}, \quad F=\mu_{F}+\epsilon_{F}
$$

where $\mu_{F}$ and $\mu_{W}$ are the noise free complex fat and water measurements, $\epsilon_{W} \sim N\left(0, \sigma^{2}\right)+i N\left(0, \sigma^{2}\right)$ and $\epsilon_{F} \sim N\left(0, \sigma^{2}\right)+i N\left(0, \sigma^{2}\right)$. Given this model the true fat fraction is $\eta_{\text {true }}=\frac{\left|\mu_{F}\right|}{\left|\mu_{F}\right|+\left|\mu_{W}\right|}$.

A signal taken from an MRI scan is complex and taking the magnitude of the signal allows us to analyze the intensity of the pixel the signal represents. The magnitude of the signals $(|F|$ and $|W|)$ follow a Rician distribution [3]. However, when the signal-to-noise ratio (SNR) (mean to standard deviation ratio) is sufficiently large (i.e., $\frac{\mu}{\sigma}>3$ ), the fat and water magnitudes are reasonably approximated by a normal distribution restricted to non-negative values [3].

\section{Methods}

\subsection{Analytical}

We simplified the probability model for the fat fraction by assuming the SNR was sufficiently high such that the fat and water magnitudes follow a normal distribution. We let $X=|F|$ and $Y=|W|$. Assuming that $X \sim N\left(\mu_{x}, \sigma_{x}^{2}\right)$ and $Y \sim N\left(\mu_{y}, \sigma_{y}^{2}\right)$, we used the method of bivariate transformations [4] to find the

joint distribution of $U=\frac{X}{X+Y}$ and $V=X+Y$, and then obtained the marginal distribution of $U$. The results for $X, Y \sim N(0,1)$ were published in a student-reviewed publication [5].

\section{$2.2 \quad$ Numerical}

The analytic expression for the distribution of the fat fraction when the fat and water magnitudes follow a Rician distribution is still unknown. Numerical simulations provided us with intuitive ideas and allowed us to explore different properties of the probability density function (pdf) of the fat fraction. We utilized Graphical User Interfaces (GUIs) to visualize the Monte Carlo simulations, used to verify analytical results, and as an aid in optimization. Our first GUI, used to verify analytic results, allows the user to control the sample size and the mean and variance for the fat and water components. Along with plotting normalized histograms for the fat and water components, the GUI plots a histogram for the fat fraction, where the true value is identified. The true fat fraction cannot be determined in clinical settings. However, since we controlled the parameters, we were able to use the GUI to determine how closely our estimation procedure estimated the true fat fraction.

We quantified the accuracy of our fat-fraction estimate by computing the sample mean squared error 
$(\widehat{\mathrm{MSE}})$. The sample MSE is the average of the square of the error, given by

$$
\widehat{\mathrm{MSE}}=\frac{1}{n} \sum_{i=0}^{n}\left(y-\hat{y}_{i}\right)^{2}
$$

where $y$ is the true fat fraction, $\hat{y}$ is obtained from simulation, and $n$ is the total number of simulated observations. Note, that when $\widehat{\mathrm{MSE}}=0$ the estimator of the fat fraction $(\hat{y})$ predicts the true value $(y)$ of the fat fraction perfectly.

By design, the MRI scanner allows the user to adjust the variances of the signals. This creates a trade-off between the variances [6]. This trade-off depends on the imaging parameters in a complicated way, and thus to simplify our analysis, we chose the relationship between the variances to be $\sigma_{F}^{2}+\sigma_{W}^{2}=1$. While this is not the actual relationship between the variances, it is a simplified approach that can give us an idea of how we can change the parameters to obtain a more accurate fat-fraction estimate by minimizing the sample MSE [6]. Our second GUI allows the user to input values for the means of the fat and water, and based on the chosen relationship, explores the effect different values for the variances of fat and water have on the sample MSE. We want to find the values of the variances that result in the lowest sample MSE, because they would tell us what values to put on the MRI scanner to obtain the best estimate of the fat fraction.

\section{Results}

\subsection{Analytical}

For our derivation, we make a few assumptions of the signal $X=|F|$ and $Y=|W|$ obtained from an MRI. We assume the $|F|$ and $|W|$ signals are independent. In reality, this is only approximately true. In addition, we assume the $\operatorname{SNR}\left(\frac{\mu}{\sigma}>3\right)$ and thus water and fat magnitudes approximately follow the following distribution [3],

$$
f_{X}\left(x ; \mu_{x}, \sigma_{x}\right)=\frac{1}{\sqrt{2 \pi} \sigma_{x}} e^{-\left(x-\sqrt{\mu_{x}^{2}+\sigma_{x}^{2}}\right)^{2} / 2 \sigma_{x}^{2}} \text { for } x \geq 0
$$

Hence, the probability density function of fat fraction $\left(U=\frac{X}{X+Y}\right)$ with high SNR is,

$$
f_{U}(u)=e^{\frac{-C}{2 \sigma_{x}^{2} \sigma_{y}^{2}}+\frac{\beta^{2}}{8 \alpha \sigma_{x}^{2} \sigma_{y}^{2}}}\left[\frac{\sigma_{x} \sigma_{y}}{2 \pi \alpha} \exp \left\{-\frac{\beta^{2}}{8 \alpha \sigma_{x}^{2} \sigma_{y}^{2}}\right\}-\frac{\beta}{4 \sqrt{2 \pi} \alpha^{3 / 2}} \operatorname{erfc}\left(\frac{\beta}{2 \sqrt{2} \sigma_{x} \sigma_{y} \alpha^{1 / 2}}\right)\right]
$$

where

$$
\begin{aligned}
\alpha(u) & =\left(\sigma_{x}^{2}+\sigma_{y}^{2}\right) u^{2}-2 \sigma_{x}^{2} u+\sigma_{x}^{2}, \\
\beta(u) & =2 \sigma_{x}^{2} B(u-1)-2 \sigma_{y}^{2} A u, \\
C & =\sigma_{x}^{2} B^{2}+\sigma_{y}^{2} A^{2}
\end{aligned}
$$

and $A=\sqrt{\mu_{x}^{2}+\sigma_{x}^{2}}$ and $B=\sqrt{\mu_{y}^{2}+\sigma_{y}^{2}}$. The analytic pdf was verified through Monte Carlo experiments, shown in Figure 2, The details of this derivation are included in Appendix A. 


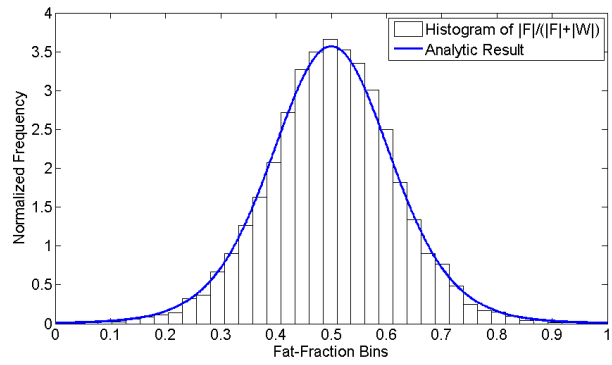

(a)

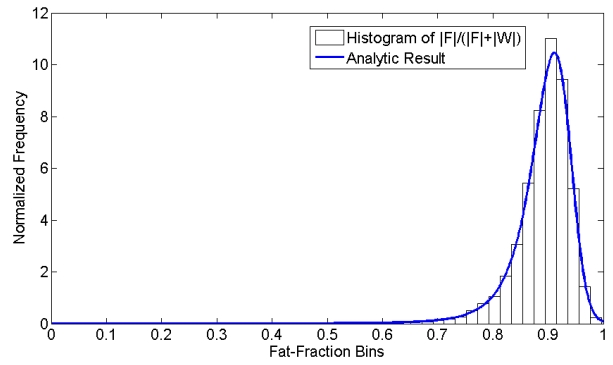

(b)

Figure 2: Our analytical result of the probability density function of the fat fraction (Eqn. 2 verified with Monte Carlo simulations: (a) represents $|F|$ and $|W|$ with $\mu_{f}=3, \sigma_{f}=1$ and $\mu_{w}=3, \sigma_{w}=1$ (fat fraction $=$ $0.5, \mathrm{SNR}=3$ ), and (b) represents $|F|$ and $|W|$ with $\mu_{f}=90, \sigma_{f}=30$ and $\mu_{w}=10, \sigma_{w}=10 / 3$ (fat fraction $=0.9, \mathrm{SNR}=3)$. In both of these simulations we used a sample size of 10,000.

We also explored the accuracy of the distribution of the fat fraction where the $|F|$ and $|W|$ have a low SNR $\left(\frac{\mu}{\sigma}<3\right)$, see Figure 3 The $|F|$ and $|W|$ only follow a normal distribution at high SNR. Thus, the analytical distribution best describes the data at high SNR.

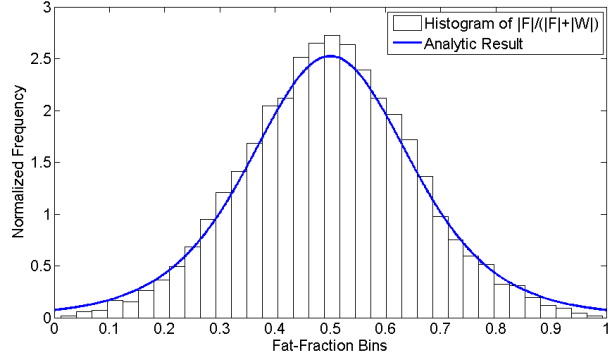

(a)

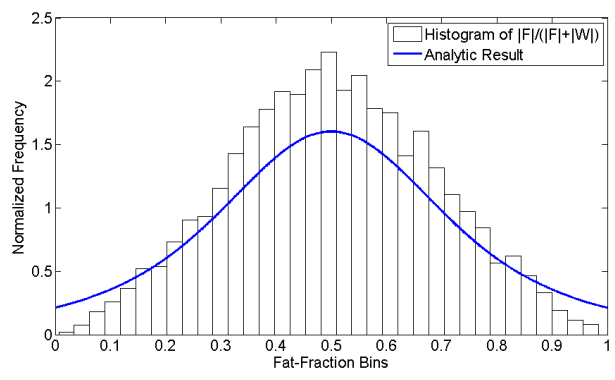

(b)

Figure 3: Our analytical result of the fat fraction pdf derived for high SNR (Eqn. 2) but with data at low SNR. From left to right we decrease the SNR of the $|W|$ and $|F|$ components. Each of the components $|F|$ and $|W|$ has a Rician distribution (a) represents $|F|$ and $|W|$ with $\mu_{f}=90, \sigma_{f}=45$ and $\mu_{w}=90, \sigma_{w}=45$ (fat fraction $=0.5, \mathrm{SNR}=2$ ) (b) represents $|F|$ and $|W|$ with $\mu_{f}=90, \sigma_{f}=90$ and $\mu_{w}=90, \sigma_{w}=90$ (fat fraction $=0.5, \mathrm{SNR}=1)$. As the SNR decreases we see the analytic distribution becomes less accurate. In both of these simulations we used a sample size of 10,000 .

\subsection{Numerical}

Monte Carlo simulations allowed us to explore the pdf of the fat fraction without needing to make the normal approximation, which is specially useful at low SNR. Our goals were to understand when our current estimation techniques gave good estimates and to optimize the variances of the fat and water components in order to obtain an accurate estimate of the fat fraction. We chose the relationship between the variances to always be $\sigma_{F}^{2}+\sigma_{W}^{2}=1$ and looked at two different cases. 


\subsubsection{Case 1: Equal Means}

When the means of the fat and the water are set to be equal to each other, the results with the lowest sample MSE are obtained when the variances of the fat and water are relatively equal, at about 0.5 (Figure 4). We verified these results using the histograms and were able to understand the behavior of the fat-fraction distribution with equal means and variances (Figure 5 ).

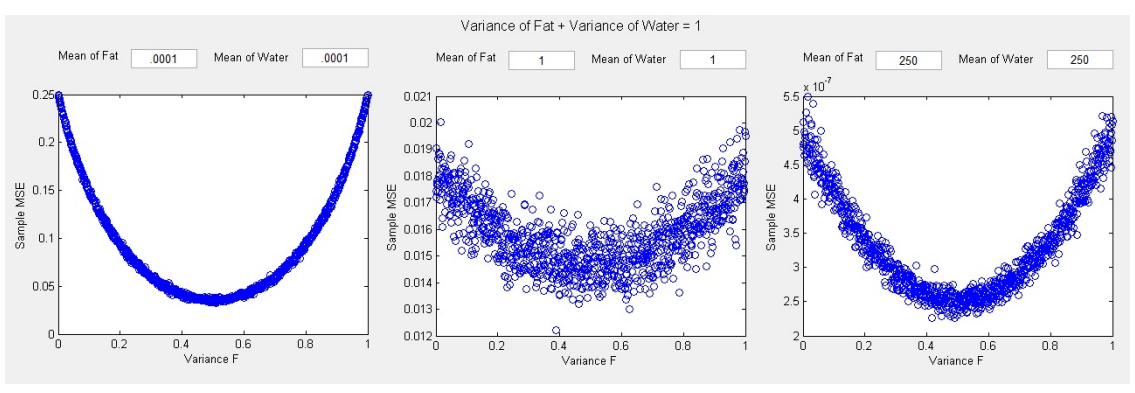

Figure 4: For values of the mean of fat equal to the mean of water, the lowest sample MSE is obtained when the variances are approximately equal. Note that in MRI the signal has arbitrary units. For this simulation we used a sample size of 1000 .

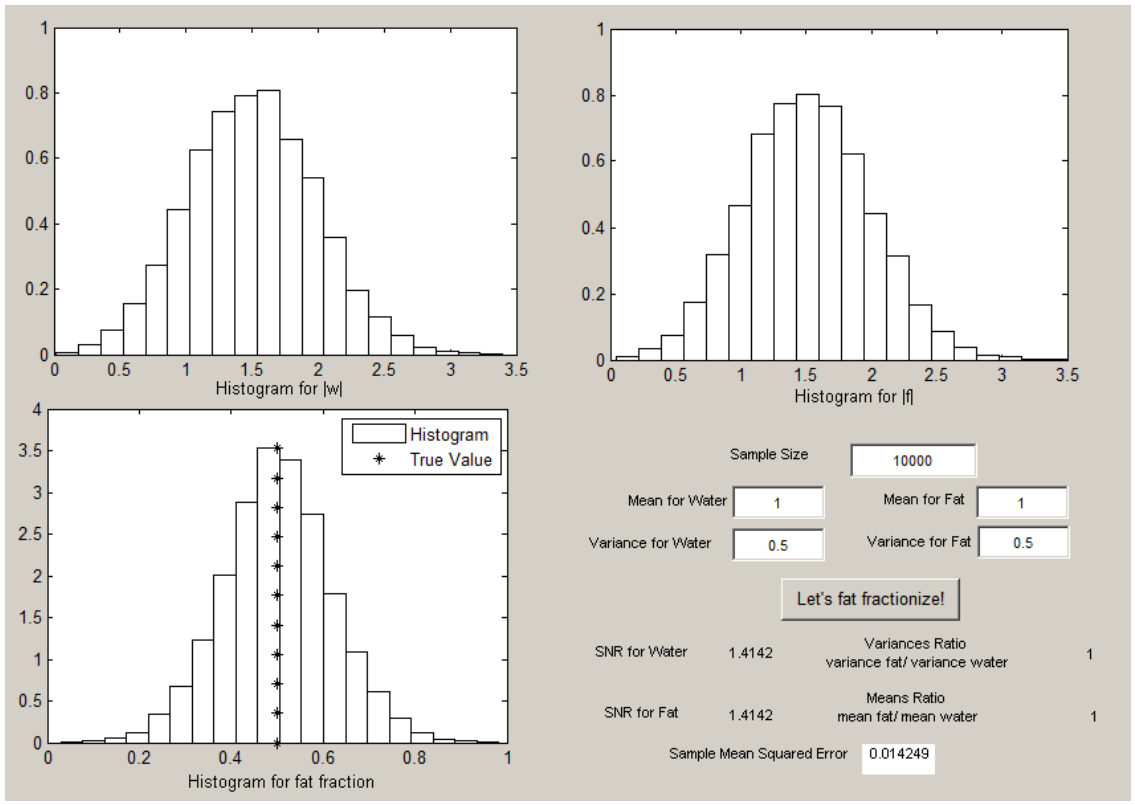

Figure 5: Corresponding to the MSE optimization in Figure 4 the means and variances of the fat and water are equal (with $\mathrm{SNR}=\sqrt{2}$ ). Notice the fat fraction is estimated with high accuracy $(\widehat{\mathrm{MSE}} \approx 0.0142$ ), and the true value of the fat fraction $\left(^{*}\right)$ is at the center of the histogram. 


\subsubsection{Case 2: One Mean is a Tenth of the Other}

When the mean of one component is set to be one-tenth of the mean of the other, the results with the lowest sample MSE are obtained when the variance of the component with the smaller mean (i.e., the fat variance) is minimized (Figure 6). This is an important result because in clinical settings it is common for the mean of the fat to be one-tenth of the mean of the water [2].
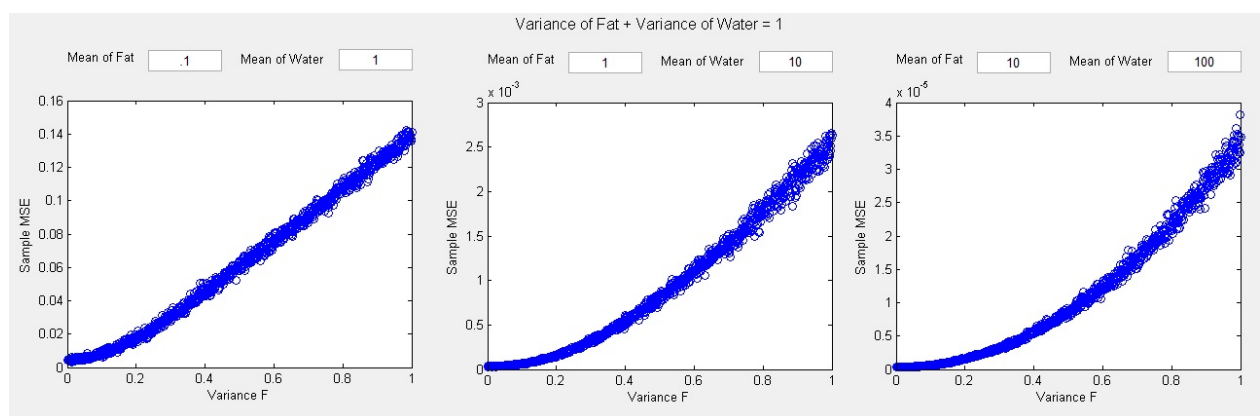

Figure 6: When the mean of the fat is one-tenth of the mean of the water, the fat component must have a very low variance in order to obtain the lowest sample MSE. For this simulation we used a sample size of 1000.

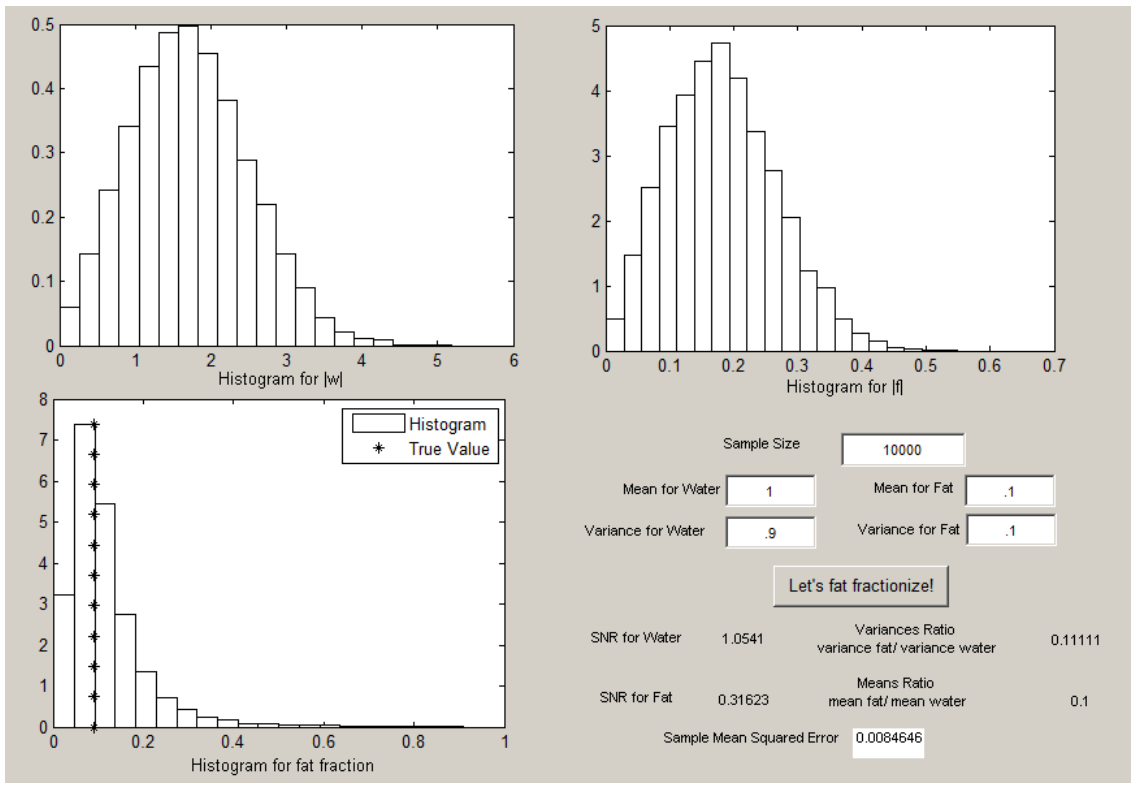

Figure 7: When the mean of fat is one-tenth of the mean of water, and the variance for the fat is low, while the variance of the water is high, the fat fraction is estimated with high accuracy $(\widehat{\mathrm{MSE}} \approx 0.00846)$. 


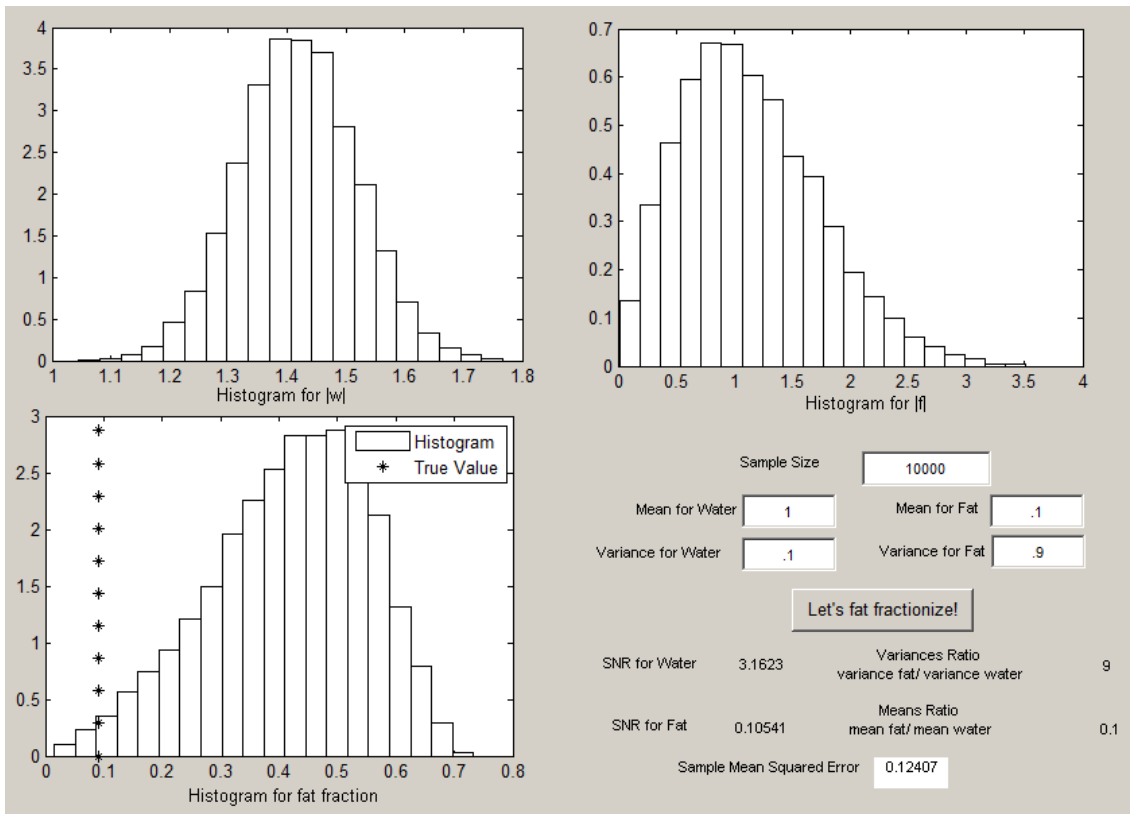

Figure 8: When the mean of fat is one-tenth of the mean of water, and the variance for the fat is high, while the variance for the water is low, the fat fraction is estimated with low accuracy ( $\widehat{\mathrm{MSE}} \approx 0.124)$. The true fat fraction is overlaid over the histogram of the fat fraction and is far from the estimated mean.

\section{Discussion}

\subsection{Fat-Fraction Expression}

We were able to derive an expression for the fat fraction assuming the magnitudes of the fat and water followed a normal distribution. This is an important step towards finding an expression where the magnitudes of the fat and water follow a Rician distribution. Our result is only applicable for high SNR.

\subsection{Monte Carlo Simulations}

Our simulations have given us insight to the relationship between the means and variances of the fat and water components, and how they affect the accuracy of our fat-fraction estimate. We know that depending on the values of the fat and water means, the variances should be changed according to our MSE optimization. For our chosen relationship between the variances, this requires a trade off. It may be necessary to increase the noise in one parameter estimate (water or fat) in order to obtain a more accurate fat-fraction estimate. We would not have been able to predict the results from our optimization before running the simulations. We would expect that the MSE would be different for a fat fraction of 0.5 than 0.9 because fat fraction needs to be between 0 and 1 . This would lead the distribution with a fat fraction with mean 0.9 to be skewed but one with mean 0.5 could be symmetric. This, however, would not allow us to predict how the MSE would change with the changes in the variances of the water and fat before running the simulations.

In Figure 7, we set the mean of the fat to be one-tenth of the mean of the water, and the variances to 
correspond with the results of our MSE optimization. The fat and water magnitude histograms appear to follow a Rician distribution and the actual fat fraction is very close to the mean of our simulation. This can be seen numerically as the sample MSE is very low at 0.00846. As optimizing the MSE is a goal of our project, this is the type of result we would like to see.

In Figure 8, we keep the parameters for mean of the fat to be one-tenth of the mean of the water, but change the variances to go against our MSE optimization results. The importance in this case is that the actual fat fraction varies significantly from our simulated fat fraction. With these parameters, it does not appear that our fat-fraction simulation estimates the true fat fraction very well. This is further verified numerically, since the sample MSE is larger at 0.124 . We would have expected our estimate of the fat fraction to be worse for low SNR but this MSE allows us to quantify by how much.

As our fat-fraction simulations represent the industry standard in estimating the fat fraction, this result is alarming. This shows that the current estimates for fat fraction used for diagnosis of disease, under certain circumstances are inaccurate. Results are exact mirror images when we switch means and have the mean of water be one-tenth of the mean of the fat.

Our results show that our estimated value for the fat fraction does not consistently predict the true fat fraction with accuracy (at low SNR). Since our simulations were run in the same way the fat fraction is currently estimated, it is apparent that the industry standard for estimating the fat fraction at low SNR requires a new approach that yields a more accurate fat-fraction estimate.

\subsection{Future Work}

As our research continues, we have plans for both the analytic and numerical aspects of the project. Analytically, we will work towards finding an expression for the distribution of the fat fraction, where the water and fat magnitudes follow a Rician distribution [3]. Numerically, we will explore a more realistic relationship between the variances of the fat and water components in an attempt to understand their effect on the fat-fraction estimate. We hope to gain sufficient understanding to enable us to explore a more clinically accurate model. We also hope to use approximate methods for the variance of functions of random variables to explain some of the results, for example, why the MSE of the fat fraction is less when the variance of the fat is small when the fat fraction is $1 / 10$. Finally, we will use the theory of maximum likelihood estimation (MLE) to determine whether there is a better estimator for the fat fraction. The MSE of the MLE at high SNR could help us connect the analytic results in this paper with the numerical results. A more general MLE (computed numerically) could provide a better way of estimating the fat fraction at all SNR.

\subsection{Acknowledgments}

We would like to thank the Mathematical Association of America Travel Grant (MAA), the National Science Foundation (NSF) grant DMS-03664 through a mini grant from the Center for Undergraduate Research in Mathematics (CURM) and the Louis Stokes Alliance for Minority Participation (LSAMP) for financial support. We would also like to thank Dr. Scott B. Reeder for the clinical image included in this paper. In addition, we would like to thank the reviewers for their thorough and helpful review. 


\section{Appendix A}

\section{A Derivation of the Fat-Fraction pdf at High SNR}

We make a few assumptions of the signal $|F|$ and $|W|$ obtained from an MRI.

- We assume the $|F|$ and $|W|$ signals are independent.

- We assume the SNR is sufficiently high to use the normal approximation for the Rician [3] and thus each having the following distribution in Eqn 1 .

- Note that because $|\cdot| \geq 0$, the normal approximation is restricted to non-negative values.

Suppose $X=|F|$ and $Y=|W|$ have the distribution described in Eqn 1. Then, their joint density is

$$
f_{X, Y}(x, y)=\frac{1}{2 \pi \sigma_{x} \sigma_{y}} \exp \left\{-\left[\frac{\left(x-\sqrt{\mu_{x}^{2}+\sigma_{x}^{2}}\right)^{2}}{2 \sigma_{x}^{2}}+\frac{\left(y-\sqrt{\mu_{y}^{2}+\sigma_{y}^{2}}\right)^{2}}{2 \sigma_{y}^{2}}\right]\right\} .
$$

Let $A=\sqrt{\mu_{x}^{2}+\sigma_{x}^{2}}$ and $B=\sqrt{\mu_{y}^{2}+\sigma_{y}^{2}}$, then

$$
f_{X, Y}(x, y)=\frac{1}{2 \pi \sigma_{x} \sigma_{y}} \exp \left\{-\left[\frac{(x-A)^{2}}{2 \sigma_{x}^{2}}+\frac{(y-B)^{2}}{2 \sigma_{y}^{2}}\right]\right\} \text {. }
$$

We want to find the distribution of $\frac{X}{X+Y}$.

Let $U=\frac{X}{X+Y}$ and $V=X+Y$, then $X=U V$ and $Y=V(1-U)$.

The Jacobian is

$$
J=\left[\begin{array}{cc}
\frac{\partial x}{\partial u}=v & \frac{\partial x}{\partial v}=u \\
\frac{\partial y}{\partial u}=-v & \frac{\partial y}{\partial v}=(1-u)
\end{array}\right] .
$$

Then $|J|=|v(1-u)+u v|=|v|$. The joint density of $f_{u v}(u, v)$ is using the method for a bivariate transformation of random values [4],

$$
\begin{aligned}
& =\frac{|v|}{2 \pi \sigma_{x} \sigma_{y}} \exp \left\{-\left[\frac{(u v-A)^{2}}{2 \sigma_{x}^{2}}+\frac{(v(1-u)-B)^{2}}{2 \sigma_{y}^{2}}\right]\right\}, \\
& =\frac{|v|}{2 \pi \sigma_{x} \sigma_{y}} \exp \left\{-\frac{1}{2 \sigma_{x}^{2} \sigma_{y}^{2}}\left[\sigma_{y}^{2}(u v-A)^{2}+\sigma_{x}^{2}(v(1-u)-B)^{2}\right]\right\} .
\end{aligned}
$$

Expanding the quantities in the exponential,

$$
\begin{aligned}
\sigma_{y}^{2}(u v-A)^{2} & =\sigma_{y}^{2}\left(u^{2} v^{2}-2 u v A+A^{2}\right), \\
\sigma_{x}^{2}(v(1-u)-B)^{2} & =\sigma_{x}^{2}\left((1-u)^{2} v^{2}-2(1-u) v B+B^{2}\right)=\sigma_{x}^{2}\left(\left(u^{2}-2 u+1\right) v^{2}-2 v B+2 u v B+B^{2}\right) .
\end{aligned}
$$

Then the exponent is equal to:

$$
-\frac{1}{2 \sigma_{x}^{2} \sigma_{y}^{2}}\left[\left(\sigma_{x}^{2}+\sigma_{y}^{2}\right) u^{2}-2 \sigma_{x}^{2} u+\sigma_{x}^{2}\right] v^{2}+\left[2 \sigma_{x}^{2} B(u-1)-2 \sigma_{y}^{2} A u\right] v+\sigma_{x}^{2} B^{2}+\sigma_{y}^{2} A^{2} .
$$


Let

$$
\begin{aligned}
\alpha(u) & =\left(\sigma_{x}^{2}+\sigma_{y}^{2}\right) u^{2}-2 \sigma_{x}^{2} u+\sigma_{x}^{2}, \\
\beta(u) & =2 \sigma_{x}^{2} B(u-1)-2 \sigma_{y}^{2} A u, \text { and } \\
C & =\sigma_{x}^{2} B^{2}+\sigma_{y}^{2} A^{2} .
\end{aligned}
$$

For a joint density $f_{U, V}(u, v)$ we get,

$$
=\frac{|v|}{2 \pi \sigma_{x} \sigma_{y}} \exp \left\{-\frac{1}{2 \sigma_{x}^{2} \sigma_{y}^{2}}\left[\alpha v^{2}+\beta v+C\right]\right\} .
$$

To find the probability density function of the fat fraction, we integrate the joint density function with respect to $v \in(0, \infty)$ (i.e. the marginal distribution of $u$ ),

$$
\begin{aligned}
f(u)=\int_{-\infty}^{\infty} f(u, v) d v & =\int_{0}^{\infty} \frac{|v|}{2 \pi \sigma_{x} \sigma_{y}} \exp \left\{-\frac{1}{2 \sigma_{x}^{2} \sigma_{y}^{2}}\left[\alpha v^{2}+\beta v+C\right]\right\} d v, \\
& =\frac{e^{\frac{-C}{2 \sigma_{x}^{2} \sigma_{y}^{2}}}}{2 \pi \sigma_{x} \sigma_{y}} \int_{0}^{\infty} v \exp \left\{-\frac{1}{2 \sigma_{x}^{2} \sigma_{y}^{2}}\left[\alpha v^{2}+\beta v\right]\right\} d v, \\
& =\frac{e^{\frac{-C}{2 \sigma_{x}^{2} \sigma_{y}^{2}}}}{2 \pi \sigma_{x} \sigma_{y}} \int_{0}^{\infty} v \exp \left\{-\frac{\alpha}{2 \sigma_{x}^{2} \sigma_{y}^{2}}\left[\left(v+\frac{\beta}{2 \alpha}\right)^{2}-\frac{\beta^{2}}{4 \alpha^{2}}\right]\right\} d v, \\
& =\frac{e^{\frac{-C}{2 \sigma_{x}^{2} \sigma_{y}^{2}}+\frac{\beta^{2}}{8 \alpha \sigma_{x}^{2} \sigma_{y}^{2}}}}{2 \pi \sigma_{x} \sigma_{y}} \int_{0}^{\infty} v \exp \left\{-\frac{\alpha}{2 \sigma_{x}^{2} \sigma_{y}^{2}}\left[\left(v+\frac{\beta}{2 \alpha}\right)^{2}\right]\right\} d v .
\end{aligned}
$$

We then let $w=v+\frac{\beta}{2 \alpha}, d w=d v\left(v=w-\frac{\beta}{2 \alpha}\right)$.

Then

$$
\begin{aligned}
f(u) & =\frac{e^{\frac{-C}{2 \sigma_{x}^{2} \sigma_{y}^{2}}+\frac{\beta^{2}}{8 \alpha \sigma_{x}^{2} \sigma_{y}^{2}}}}{2 \pi \sigma_{x} \sigma_{y}} \int_{\frac{\beta}{2 \alpha}}^{\infty}\left(w-\frac{\beta}{2 \alpha}\right) e^{-\frac{\alpha}{2 \sigma_{x}^{2} \sigma_{y}^{2}}\left(w^{2}\right)} d w, \\
& =\frac{e^{\frac{-C}{2 \sigma_{x}^{2} \sigma_{y}^{2}}+\frac{\beta^{2}}{8 \alpha \sigma_{x}^{2} \sigma_{y}^{2}}}}{2 \pi \sigma_{x} \sigma_{y}} \int_{\frac{\beta}{2 \alpha}}^{\infty}\left[w e^{-\frac{\alpha}{2 \sigma_{x}^{2} \sigma_{y}^{2}}\left(w^{2}\right)}-\left(\frac{\beta}{2 \alpha}\right) e^{-\frac{\alpha}{2 \sigma_{x}^{2} \sigma_{y}^{2}}\left(w^{2}\right)}\right] d w, \\
& =\frac{e^{\frac{-C}{2 \sigma_{x}^{2} \sigma_{y}^{2}}+\frac{\beta^{2}}{8 \alpha \sigma_{x}^{2} \sigma_{y}^{2}}}}{2 \pi \sigma_{x} \sigma_{y}}[\underbrace{\int_{\frac{\beta}{2 \alpha}}^{\infty} w e^{-\frac{\alpha}{2 \sigma_{x}^{2} \sigma_{y}^{2}}\left(w^{2}\right)} d w}_{\text {integral 1 }}-\underbrace{\int_{\frac{\beta}{2 \alpha}}^{\infty}\left(\frac{\beta}{2 \alpha}\right) e^{-\frac{\alpha}{2 \sigma_{x}^{2} \sigma_{y}^{2}}\left(w^{2}\right)} d w}_{\text {integral 2 }} .
\end{aligned}
$$

We solve each integral separately. We solve, Integral 1:

$$
\int_{\frac{\beta}{2 \alpha}}^{\infty} w e^{-\frac{\alpha}{2 \sigma_{x}^{2} \sigma_{y}^{2}}\left(w^{2}\right)} d w .
$$

Let $t=-\frac{\alpha}{2 \sigma_{x}^{2} \sigma_{y}^{2}}\left(w^{2}\right)$, then $d t=-\frac{\alpha}{\sigma_{x}^{2} \sigma_{y}^{2}} w d w$.

$$
\int_{\frac{\beta}{2 \alpha}}^{\infty} w e^{-\frac{\alpha}{2 \sigma_{x}^{2} \sigma_{y}^{2}}\left(w^{2}\right)} d w=\frac{\sigma_{x}^{2} \sigma_{y}^{2}}{\alpha} \exp \left\{-\frac{\beta^{2}}{8 \alpha \sigma_{x}^{2} \sigma_{y}^{2}}\right\} .
$$

We solve, Integral 2:

$$
\int_{\frac{\beta}{2 \alpha}}^{\infty} \frac{\beta}{2 \alpha} \exp \left\{-\frac{\alpha}{2 \sigma_{x}^{2} \sigma_{y}^{2}} w^{2}\right\} d w .
$$


Rewrite the integral, we have

$$
\int_{\frac{\beta}{2 \alpha}}^{\infty} \frac{\beta}{2 \alpha} \exp \left\{-\left(\frac{\sqrt{\alpha} w}{\sqrt{2} \sigma_{x} \sigma_{y}}\right)^{2}\right\} d w
$$

Let $r=\frac{\sqrt{\alpha} w}{\sqrt{2} \sigma_{x} \sigma_{y}}$. Then $d r=\frac{\sqrt{\alpha}}{\sqrt{2} \sigma_{x} \sigma_{y}} d w$. Thus,

$$
=\frac{\beta \sqrt{2} \sigma_{x} \sigma_{y}}{2 \alpha^{3 / 2}} \int_{\frac{\beta}{2 \sqrt{2} \sigma_{x} \sigma_{y} \alpha^{1 / 2}}}^{\infty} e^{-r^{2}} d r .
$$

Now substitute integral 1 and integral 2 back to $f(u)$, we obtain,

$$
\begin{aligned}
f(u) & =\frac{e^{\frac{-C}{2 \sigma_{x}^{2} \sigma_{y}^{2}}+\frac{\beta^{2}}{8 \alpha \sigma_{x}^{2} \sigma_{y}^{2}}}}{2 \pi \sigma_{x} \sigma_{y}}\left[\frac{\sigma_{x}^{2} \sigma_{y}^{2}}{\alpha} \exp \left\{-\frac{\beta^{2}}{8 \alpha \sigma_{x}^{2} \sigma_{y}^{2}}\right\}-\frac{\beta \sqrt{2} \sigma_{x} \sigma_{y}}{2 \alpha^{3 / 2}} \int_{\frac{\beta}{2 \sqrt{2} \sigma_{x} \sigma_{y} \alpha^{1 / 2}}}^{\infty} e^{-r^{2}} d r\right], \\
& =e^{\frac{-C}{2 \sigma_{x}^{2} \sigma_{y}^{2}}+\frac{\beta^{2}}{8 \alpha \sigma_{x}^{2} \sigma_{y}^{2}}}\left[\frac{\sigma_{x} \sigma_{y}}{2 \pi \alpha} \exp \left\{-\frac{\beta^{2}}{8 \alpha \sigma_{x}^{2} \sigma_{y}^{2}}\right\}-\frac{\beta}{4 \sqrt{2 \pi} \alpha^{3 / 2}} \frac{2}{\sqrt{\pi}} \int_{\frac{\beta}{2 \sqrt{2} \sigma_{x} \sigma_{y} \alpha^{1 / 2}}}^{\infty} e^{-r^{2}} d r\right], \\
& =e^{\frac{-C}{2 \sigma_{x}^{2} \sigma_{y}^{2}}+\frac{\beta^{2}}{8 \alpha \sigma_{x}^{2} \sigma_{y}^{2}}}\left[\frac{\sigma_{x} \sigma_{y}}{2 \pi \alpha} \exp \left\{-\frac{\beta^{2}}{8 \alpha \sigma_{x}^{2} \sigma_{y}^{2}}\right\}-\frac{\beta}{4 \sqrt{2 \pi} \alpha^{3 / 2}} \operatorname{erfc}\left(\frac{\beta}{2 \sqrt{2} \sigma_{x} \sigma_{y} \alpha^{1 / 2}}\right)\right] .
\end{aligned}
$$

Note that the Error function is defined as,

$$
\operatorname{erf}(x)=\frac{2}{\sqrt{\pi}} \int_{0}^{x} e^{-t^{2}} d t
$$

Then, the Complementary error function is defined as,

$$
\operatorname{erfc}(x)=1-\operatorname{erf}(x)=\frac{2}{\sqrt{\pi}} \int_{x}^{\infty} e^{-t^{2}} d t .
$$

Hence we found the probability density function of the fat fraction when signal-to-noise ratio is high given by Eqn 11:

$$
f_{U}(u)=e^{\frac{-C}{2 \sigma_{x}^{2} \sigma_{y}^{2}}+\frac{\beta^{2}}{8 \alpha \sigma_{x}^{2} \sigma_{y}^{2}}}\left[\frac{\sigma_{x} \sigma_{y}}{2 \pi \alpha} \exp \left\{-\frac{\beta^{2}}{8 \alpha \sigma_{x}^{2} \sigma_{y}^{2}}\right\}-\frac{\beta}{4 \sqrt{2 \pi} \alpha^{3 / 2}} \operatorname{erfc}\left(\frac{\beta}{2 \sqrt{2} \sigma_{x} \sigma_{y} \alpha^{1 / 2}}\right)\right] .
$$

\section{References}

[1] Reeder S., Robson P., Yu H., Shimakawa A., Hines C., McKenzie H., Brittain J. Quantification of Hepatic Steatosis With MRI: The Effects of Accurate Fat Spectral Modeling. Journal of Magnetic Resonance Imaging. 29:1332-1339 (2009).

[2] Reeder S., Sirlin B. Quantification of Liver Fat with Magnetic Resonance Imaging. Magnetic Resonance Imaging Clinics of North America. 18:337-357 (2010).

[3] Gudbjartsson, H. and Patz, Samuel. The Rician Distribution of Noisy MRI Data. Magnetic Resonance in Medicine. 34:910-914 (1995).

[4] DeGroot, M. and Schervish, M. Probability and Statistics, 3rd ed. Addison-Wesley (2002).

[5] Calder A.M., Ellis A. E., Huang, L.H., and Park, K. Statistical Modeling of the Fat Fraction in Magnetic Resonance Imaging (MRI). DIMENSIONS - The Journal of Undergraduate Research in Natural Sciences and Mathematics. 13:114-123 (2001).

[6] Pineda, A. R., Reeder, S. B., Wen, Z., and Pelc, N. J. Cramer-Rao Bounds for Three-Point Decomposition of Water and Fat. Magnetic Resonance in Medicine. 54:625-635 (2005). 